\title{
An Update on the Outcomes of Patients Treated with Urolift for Benign Prostatic Hyperplasia
}

\author{
Justin Loloi' \\ Nathan Feiertag ${ }^{2}$ \\ Kripali Gautam ${ }^{2}$ \\ Pedro Maria' \\ 'Department of Urology, Montefiore \\ Medical Center, Bronx, NY, USA; ${ }^{2}$ Albert \\ Einstein College of Medicine, Bronx, \\ NY, USA
}

\begin{abstract}
Benign prostatic hyperplasia (BPH) is a ubiquitous urologic disease affecting aging men. Patients often experience bothersome lower urinary tract symptoms (LUTS) that warrant urologic evaluation and management. Routinely, patients are initially treated with medical therapies with the goal of both relaxing the bladder neck and shrinking the prostate in order to relieve obstruction secondary to prostatic enlargement. Transurethral resection of the prostate (TURP) serves as a first-line surgical intervention in those who fail medical therapy. Recently, novel minimally invasive surgical techniques for BPH management have emerged. Of these, prostatic urethral lift (PUL or Urolift) has gained attention given its presumed effectiveness and minimal risk of sexual side effects when compared to the standard TURP. The purpose of this review is to describe past and current trends in the implementation of PUL for BPH and to highlight important outcomes.
\end{abstract}

Keywords: benign, prostate, minimally-invasive, urethral, urinary, sexual

\section{Introduction}

Benign prostatic hyperplasia (BPH) is a common urologic disease in which the prostatic tissue grows, often causing bothersome lower urinary tract symptoms ${ }^{1,2}$ that may have a significant negative effect on quality of life. ${ }^{3}$ Typically, management begins with medical therapies such as alpha-adrenergic antagonists to relax the bladder neck and 5-alpha reductase inhibitors to decrease the size of the prostate. For those who fail medical management, a wide array of surgical interventions are available for managing symptoms and improving quality of life. ${ }^{4-6}$

Classically, the gold-standard surgical management of BPH was transurethral resection of the prostate (TURP). However, there exists a considerable risk of erectile and ejaculatory dysfunction. ${ }^{7}$ Consequently, over the years, various surgical methods for the treatment of BPH have been adopted. In addition to ablative therapies such as holmium laser enucleation of the prostate (HoLEP) and greenlight/photoselective vaporization of the prostate, non-ablative measures such as water vapor thermal therapy (Rezum) and, more recently, the prostatic urethral lift (PUL) have gained popular attention. ${ }^{8}$

PUL represents an innovative, minimally invasive therapeutic option in the treatment of symptomatic $\mathrm{BPH} .{ }^{9}$ Rather than ablating or cavitating the prostate gland, the PUL technology aims to mechanically manipulate the prostate as a means of creating an open channel thus allowing for low-resistance passage of urine. During the procedure, adjustable implants are deployed and serve to retract the obstructing lobes of the prostate..$^{10}$ Due to its non-ablative nature, an advantage of
Correspondence: Pedro Maria Department of Urology, Montefiore Medical Center, Bronx, NY, 10467, USA Tel + I 7189204531

Email pmaria@montefiore.org 
PUL is its preservation of sexual function ${ }^{11-13}$ and potential application under the setting of local anesthesia. ${ }^{14}$ The importance of this sentiment cannot be understated as sexual function has been shown to be predictive for overall quality of life, overall health perception, physical, psychological, social relations, and environmental domains using a World Health Organization Quality of Life Index (WHOQOL-bref). ${ }^{15}$

Patient selection is paramount to the success of the procedure. Although there is no definitive criteria for those warranting PUL, patients are typically age 50 and above with medium-sized prostates ( $20-70 \mathrm{cc}$ on ultrasound) and symptomatic lower urinary tract symptoms in the setting of decreased urinary flow ( $\mathrm{Q} \max <15 \mathrm{~mL}$ ). An important exclusion criteria typically used is an obstructing median lobe. ${ }^{9}$

Despite the appealing aspects of the PUL procedure in the management of $\mathrm{BPH}$, there is a relative paucity of studies delineating outcomes. The aim of this paper is to provide a review of the literature surrounding the implementation of PUL and an update on the outcomes of patients treated with PUL for BPH.

\section{The L.I.F.T. Trial and Follow-Up}

In 2013, the first multicenter, randomized blinded trial of PUL for the treatment of LUTS secondary to BPH was published, with the goal of determining the safety and efficacy of this new technology. The L.I.F.T. study by Roehrborn et al represents a key work describing outcomes in PUL. The study consisted of 19 centers across the world (14 in the US, 3 in Australia, and 2 in Canada) evaluating several outcomes including LUTS severity via International Prostate Symptom Score (IPSS), quality of life, BPH impact index (BPHII), Qmax, sexual function, and adverse events. ${ }^{16}$ Table 1 lists the inclusion and exclusion criteria utilized in the study (Table 1). Overall, 206 participants were randomised (PUL: $n=140$; sham: $n=66$ ). The sham control procedure mimicked the experience of undergoing a PUL by having the surgeons blinding the participants with a surgical drape, calling for/opening procedural devices, and deploying a disposable biopsy device to simulate PUL device sounds. The primary endpoint was comparison of AUA symptom index (AUASI) reduction at 3 months. The PUL subjects were evaluated at 1-year postoperative to assess for LUTS, peak urinary flow rate, sexual function and quality of life.
Table I Inclusion and Exclusion Criteria for the L.I.F.T. Study

\begin{tabular}{|l|l|}
\hline Inclusion Criteria & $\begin{array}{l}\text { Exclusion } \\
\text { Criteria }\end{array}$ \\
\hline Age > or equal to 50 years & $\begin{array}{l}\text { Obstructive } \\
\text { median lobe }\end{array}$ \\
\hline $\begin{array}{l}\text { International Prostate Symptom Score > or } \\
\text { equal to } 13\end{array}$ & $\begin{array}{l}\text { Active urinary } \\
\text { tract infection }\end{array}$ \\
\hline $\begin{array}{l}\text { Peak flow rate }(\text { Qmax })<\text { or equal to } 12 \mathrm{~mL} / \mathrm{s} \\
\text { with a } 125 \mathrm{~mL} \text { voided volume }\end{array}$ \\
\hline $\begin{array}{l}30 \mathrm{cc} \text { to } 80 \mathrm{cc} \text { volume prostate measured by } \\
\text { transrectal ultrasound }\end{array}$ \\
\hline
\end{tabular}

At 3 months, the mean reduction of AUASI in the PUL cohort was $88 \%$ greater than it was in the sham control and PUL therapeutic effects were significantly better than control with regard to Qmax, quality of life and BPHII. At 3 months, the sham cohort were given the option for crossover active treatment after unblinding. Fifty-three of the $66(80 \%)$ sham control participants received the PUL treatment. Additionally, with regard to safety and tolerability of the procedure, adverse events such as postoperative dysuria, hematuria, pain/discomfort and urgency were typically mild to moderate and resolved within two weeks. Notably, there were no instances of ejaculatory or erectile dysfunction. In $94 \%$ of the patients, a cystoscopy was done at 12 months and revealed no strictures, mild inflammation in one patient and edema in five patients. Encrustation was found in only $2.1 \%$ of the implants. Retreatment rate at one year was $5 \%$ in this study. Based on these findings, the authors suggested that PUL can provide efficacious treatment in an outpatient setting under local anesthesia with minimal morbidity and zero alterations of sexual function. Improvement in AUASI by $25 \%$ can be noticed at two weeks, continue to improve up until the 3-month mark, and remain stable at 1 -year postoperative time.

Five year results of the prospective, randomized controlled prostatic urethral L.I.F.T. study were recently published. ${ }^{17}$ At the 5-year interval, 104 of the 140 participants who received the PUL treatment were available for data collection $(74.3 \%)$. The reasons for disinvolvement included: 21 subjects lost to follow-up (16\%), 9 subjects receiving additional TURP/laser therapy (9\%), 7 subjects 
being retreated $(6 \%), 9$ subjects died due to unrelated causes (7\%), 3 subjects lost to protocol deviations (2\%), and 2 subjects lost to transurethral resection of bladder neck and prostatectomy respectfully.

The LIFT study demonstrated rapid LUTS improvement beginning at the 2 week follow up that lasted throughout the 5-year post-treatment period. ${ }^{16,17}$ At the 3 month period, participants in the PUL group had an $88 \%$ greater reduction in IPSS compared to the sham control group (IPSS improvement: PUL $-11.1 \pm 7.7$, sham $-5.9 \pm$ $7.7, \mathrm{p}<0.003)$. At 5 years, the IPSS score of the PUL group continued to steadily increase although remained significantly lower than the sham group (IPSS of PUL at 5 years: $-7.85, \mathrm{p}<0.001)$. Quality of life and Qmax were also significantly improved for the PUL compared to the sham control group. Statistically significant improvements in quality of life and Qmax were durable through the 5-year period (baseline: quality of life $4.62 \pm 1.05$, Qmax $7.88 \pm 2.41 ; 5$ years: quality of life $2.54 \pm 1.76$, Qmax $11.08 \pm 4.72, p<0.001)$. Similarly, the reductions in BPHII were durable through the 5-year period (baseline BPHII: $6.92 \pm 2.79,5$ years BPHII: $3.51 \pm 3.34$ ). The PUL participants retained sexual and ejaculatory function without any sustained events for the duration of the 5-year period. Mean erectile function, measured by the International Index of Erectile Function (IIEF-5), and ejaculatory function, measured by the Male Sexual Health Questionnaire (MSHQ-EjD), were unaffected by PUL, and participants showed improvement of prior bothersome symptoms due ejaculatory dysfunction through the 5-year period. Overall, improvements in IPSS, QOL, BPHII, and Qmax after undergoing PUL were stable. A minority $(13.6 \%)$ of the original patients needed surgical retreatment. Adverse effects were mild and transient. Of note, sexual function remained intact as none of the patients developed sustained erectile dysfunction or ejaculatory dysfunction. Thus, the overall results of the L.I.F.T. study revealed the utility of the PUL technology in improving LUTS while maintaining quality of life and sexual function.

\section{Outcomes Following the L.I.F.T. Trial}

Following the L.I.F.T trial, there have been a number of studies aiming to further delineate the benefits and potential drawbacks of PUL. Table 2 outlines the major trials conducted in evaluating the use of PUL for BPH (Table 2).
Based on the evidence provided by these studies, the PUL procedure has demonstrated efficacy and safety in treating LUTS due to BPH with lateral prostatic lobe enlargement. Interestingly, despite the notion that PUL is not indicated in men with obstructive median lobes, the MedLift study aimed to put this claim to the test. This study acted as an extension to the pivotal L.I.F.T. work by including subjects with LUTS due to median lobe enlargement. As so, enrollment criteria and study assessments were identical to that of the LIFT study with follow up results occurring at $1,3,6$, and 12 months post procedure. ${ }^{18}$

During the enrollment period, 71 participants with an obstructive median lobe (OML) who were similar to the active and control arms of the L.I.F.T. study were screened and 45 chosen to undergo PUL. These subjects differed from those in the LIFT study by mean age (OML: 64 years, LIFT active: 67 years, $p=0.03$ ) and increased severity of symptoms per IPSS (OML: 24.2, LIFT active: 22.2, $\mathrm{p}=0.04)$. IPSS improvements were seen immediately at the 1 month follow-up $(59 \%, \mathrm{p}<0.0001)$ with sustainment through 12 months $(55.1 \%$, p $<0.0001)$. Similarly, quality of life and BPHII scores improved $(>60 \%$ and $>70 \%$, respectively) and maximum urinary flow improved (ranging from 90\% to 130\%) through 12 months. Results were consistent for all subjects despite variations in intravesical prostatic protrusion.

The PUL procedure for OML demonstrated safety with zero post-procedure device-related serious complications. At 12 months, no subjects required medical adjuvant therapy and only one subject (2\%) needed additional implants. Additionally, sexual function was preserved with no significant change in erectile function (IIEF-5) or ejaculatory function (MSHQ-EjD Function) and zero cases of de novo sustained sexual dysfunction were reported. Therefore, when combined with the results from the L.I.F.T. study 5 year follow-up, this work reinforces the impact and effectiveness of PUL for LUTS due to BPH in patients with either lateral lobe or median lobe obstruction.

In addition to an obstructing median lobe, an oftenmentioned limitation to the use of PUL is large-sized prostates as it is not routinely indicated in patients with a prostate size of greater than $80 \mathrm{~g}$. However, there is evidence to suggest a role for PUL in this patient population. Shah et al categorized 74 patients who underwent PUL for BPH into two groups: prostate size greater than 80 grams (23 patients) and prostate size less than 80 grams (51 patients). ${ }^{19}$ With a median follow-up time of 144 days, patients in both groups reported a significant improvement 
Table 2 Outcomes in the Key PUL Trials

\begin{tabular}{|c|c|c|}
\hline Study & Factors/Outcomes Measured & Major Results \\
\hline L.I.F.T. study ${ }^{16}$ & $\begin{array}{l}\text { Measured IPSS, QoL, Qmax, BPHII, IIEF-5, MSHQ-EjD } \\
\text { function/bother after PUL treatment for LUTS due to BPH } \\
\text { with lateral lobe obstruction }\end{array}$ & $\begin{array}{l}\text { Significant and durable improvement in IPSS, QoL, Qmax, } \\
\text { BPHII without impairment of sexual function through } 5 \\
\text { years. }\end{array}$ \\
\hline MedLift study ${ }^{18}$ & $\begin{array}{l}\text { Expanded on L.I.F.T. study design by using participants } \\
\text { treated with PUL due to BPH with median lobe } \\
\text { obstruction }\end{array}$ & $\begin{array}{l}\text { Similarly to the L.I.F.T. study, participants saw an } \\
\text { improvement in IPSS, QoL, Qmax, BPHII without } \\
\text { impairment of sexual function through I year. }\end{array}$ \\
\hline BPH6 study ${ }^{21}$ & $\begin{array}{l}\text { BPH6 index was used to measure composite of the } \\
\text { following: } \\
\text { symptom relief, quality of recovery, erectile function } \\
\text { preservation, continence preservation, and safety. } \\
\text { Additional factors measured: } \\
\text { Patient perspective } \\
\text { Quality of life } \\
\text { Sleep quality }\end{array}$ & $\begin{array}{l}\text { Compared to TURP, PUL resulted in higher quality } \\
\text { recovery, preservation of ejaculatory function, and higher } \\
\text { overall BPH6 index. TURP resulted in significantly } \\
\text { compromised urinary continence function } 2 \text { weeks and } 3 \\
\text { months following procedure. PUL resulted in superior } \\
\text { improvement of sleep compared to TURP. }\end{array}$ \\
\hline $\begin{array}{l}\text { 2-Year Outcomes of } \\
\text { a Retrospective } \\
\text { Multicenter Study }{ }^{24}\end{array}$ & $\begin{array}{l}\text { IPSS } \\
\text { QoL } \\
\text { Qmax } \\
\text { Age } \\
\text { Prostate size } \\
\text { Site of service } \\
\text { Prior prostate cancer treatment } \\
\text { Diabetic status }\end{array}$ & $\begin{array}{l}\text { Significant improvement in IPSS throughout two years } \\
\text { following PUL. Subjects with IPSS greater than or equal to } \\
\text { I } 3 \text { exhibited similar results to L.I.F.T. study. } \\
\text { Age, diabetic status, prostate volume, site of service, a prior } \\
\text { cancer therapy did not affect outcomes of PUL. Subjects } \\
\text { were older in age and less symptomatic than those included } \\
\text { in L.I.F.T. study. }\end{array}$ \\
\hline
\end{tabular}

in AUASS. Specifically, between the two groups, there was no difference in AUASS prior to or following PUL. Despite a short duration of follow-up, the results of this study suggest a potential role in implementing PUL even in those with a prostate size greater than 80 grams.

Another study evaluating longer-term results came when Rukstalis et al aimed to evaluate the 24-month effectiveness of PUL in men with LUTS secondary to $\mathrm{BPH}^{20}$ In this study, the sham patients from the L.I.F. T. trial were given crossover PUL treatment and were followed to assess urinary symptom relief, quality of life, urinary flow rate, sexual function, and adverse events. At 24 months, IPSS, quality of life, BPHII, and maximum urinary flow rate improved $36 \%, 40 \%, 54 \%$, and $77 \%$ from baseline, respectively. Each IPSS parameter improved significantly from baseline $(\mathrm{p}<0.005)$ and remained stable throughout the duration of follow-up. Adverse events were mild to moderate and resolved quickly. $8 \%$ of patients ultimately required reoperation with a TURP. Similarly to the L.I.F.T. study, there were no instances of erectile or ejaculatory dysfunction. These outcomes provided further support that PUL is associated with lasting symptomatic relief and quality of life improvements without the risk of morbidity and sexual dysfunction.

In 2017, Gratzke et al published results from the BPH6 study, a prospective, multicenter, randomized non-blinded study consisting of 80 patients from 10 European centers, comparing PUL to TURP in the treatment of LUTS attributed to $\mathrm{BPH}^{21}$ Specifically, the main objective was to establish non-inferiority of PUL compared to TURP. To this end, subjects were randomized to receive either PUL $(n=45)$ or TURP $(n=35)$. The BPH6 responder endpoint combined six validated instruments and was used to assess subjects for symptom relief, quality of recovery, preservation of continence, and preservation of erectile function.

Subjects were found to have significantly better recovery experience at 1-month follow-up after PUL compared to TURP. Post-procedure response rate was higher in the PUL arm, at $100 \%$ compared to $60.6 \%$ response rate for patients who underwent TURP. Significantly, more subjects required catheterization for greater than 24 hours following TURP ( $p$ $=0.01$ ) and patients who underwent PUL returned to preoperative activity faster (11 days post-procedure) compared 
to patients who underwent TURP (17 days post-procedure). Both PUL and TURP resulted in significant improvement of LUTS compared to baseline as determined by changes in IPSS, IPSS quality of life, BPHII, and Qmax. Both procedures resulted in preserved urinary continence. However, subjects who underwent TURP had greater improvement in IPSS, Qmax, and postvoid residual volume compared to subjects who underwent PUL $(\mathrm{p}<0.05)$. Analysis for preservation of sexual function was based on subjects' sexual health inventory for men score (SHIM) and was significantly higher in subjects who received PUL. According to the Clavien-Dindo classification system, there was no significant difference in adverse events following treatment. Both procedures had similar rates of reintervention at $6.8 \%$ for PUL and $5.75 \%$ for TURP $(\mathrm{p}>0.05)$. Subjects were also evaluated for sleep disturbances compared to subjects' baselines following PUL or TURP by use of the Jenkins Sleep Questionnaire. Only PUL was found to significantly improve sleep at 6,12 and 24 months following procedure. ${ }^{21}$

Overall, subjects in the BPH6 study experienced improvement of LUTS from baseline, health-related quality of life, and Qmax after undergoing TURP or PUL. Subjects who underwent TURP experienced significantly greater improvement in LUTS compared to subjects who underwent PUL, while patients who underwent PUL were found to have improved quality of recovery, ejaculatory function, and improvement in sleep compared the TURP arm. Follow-up at 2 weeks and 3 months following TURP resulted in significantly compromised urinary function, which was also associated with a decreased health-related quality score. Thus, the results of this study underscore the importance of tailoring clinical treatment with the individual goals of patients.

Clearly, a critical consideration in the use of PUL is patient selection. To elucidate and further characterize ideal patient selection for optimum clinical outcome, Al-Singary et al retrospectively reviewed 51 men that underwent PUL. ${ }^{22}$ Over 2 years postoperatively, the 51 men had a reported greater than 90\% success rate with improvements in Qmax, postvoid residual, IPSS, and preservation in erectile function scores. A minority of patients (19.6\%) experienced mild adverse effects such as dysuria that spontaneously resolved. Notably, four patients did not report improvement following PUL. However, overall, the findings of this study shed light on important factors related to patient selection in PUL including those with high residual volumes, weakened urinary flow, and a focus on preservation of IPSS and erectile function. ${ }^{22}$

Despite the seemingly rigid criteria for patient selection in PUL, there is evidence suggesting its versatility. A study consisting of five German departments aimed to investigate outcomes in patients treated with PUL without the strict criteria established in previous studies (only exclusion criteria here being an obstructing median lobe). ${ }^{23}$ Patients were followed for $1,6,12$, and 24 months postoperatively and were assessed for maximum urinary flow, PVR, IPSS, and quality of life. Patients were given the choice of PUL despite them being candidates for TURP; 86 of the 212 patients (41\%) elected for PUL. $38.4 \%$ of patients who ultimately proceeded with PUL had severe BPH obstruction that would have otherwise been denied PUL. Within 1 month, 74 (86\%) of patients experienced significant symptom relief and improvements in all outcome measures $(\mathrm{p}<0.001)$, this was found to be sustained during follow-up. With respect to retreatment, 11 patients $(12.8 \%)$ were retreated over the 24 months of followup. Nonetheless, the results of this study suggested that even in the setting of severe obstruction, patients who would otherwise qualify for TURP may be suitable candidates for PUL.

A more recent multicenter study aiming to provide realworld evidence of the success of PUL consisted of 1413 patients from 14 sites across the United States and Australia. $^{24}$ The purpose of this real-world retrospective (RWR) study was to compare real-world results of patients who underwent PUL from multiple sites with results observed in previous controlled clinical trials such as the L.I.F.T. trial. Subjects were included in the study if they had a baseline IPSS greater than or equal to 13 documented within 9 months prior to PUL and at least one IPSS documented within 12 months following procedure. Thereafter, subjects were further stratified into two groups, with those documented to have baseline urinary retention assigned to Group B $(n=165)$ while patients with spontaneous voiding were assigned to Group A ( $\mathrm{n}=1248)$. The study further analyzed patients from Group A using paired t-tests and $95 \%$ confidence intervals to compare mean differences and percent change from subject baseline IPSS, quality of life, and maximum urinary flow rate at 1, 3, 6, 12, and 24 months after undergoing PUL procedures. The study also analyzed adverse events, surgical retreatments, and catheterization rates in both groups. ${ }^{24}$

When compared to subjects from the L.I.F.T. study, RWR patients were older in age with lower IPSS at baseline, higher Qmax, and lower quality of life. Subjects in Group A with baseline spontaneous voiding were found to have an improvement from baseline IPSS by a minimum of 8.1 after undergoing PUL. Group A subjects had no significant difference in post-procedure scores compared to subjects in Group B with baseline urinary retention. Subjects in Group A who had a baseline IPSS greater than or equal to 13 had similar 
postprocedural symptom outcomes compared to subjects from the L.I.F.T. study. An analysis for potential differences in PUL outcomes considered age, prostate volume, site of service, prior cancer treatment, and diabetic status, all of which were found to have no significant impact. Furthermore, subjects who had undergone treatment for prostate cancer did not experience increased adverse events following PUL compared to subjects with no history of prior cancer treatment. ${ }^{24}$

This retrospective study provides insight to real-world outcomes associated with PUL in comparison to previous clinical trials that took place in highly controlled clinical settings. The study included patients with both smaller and larger prostate volumes and lower severity of symptoms compared to subjects in clinical trials. Similar outcomes between subjects in this retrospective study and previous clinical trials, despite baseline differences, allow for application of results from the L.I.F.T. study to a broader patient population that reflects clinical practice.

The focus on real-world applicability and practicality is an important consideration, particularly in a disease that tends to affect older men who may have existing comorbidities. Kim et al conducted a retrospective study of patients with BPH treated with PUL, consisting of 32 men with an average age 67 years and with significant comorbidities including diabetes mellitus (50\%), hypertension (75\%), and ischemic heart disease $(28 \%){ }^{25}$ All procedures were performed under local anesthesia using a combination of a sedative and lidocaine and patients were followed up for one year. Following PUL, patients experienced symptom relief within one week that was sustained for the one year study duration; specifically, at one week postoperatively, there were significant improvements in mean IPSS (43\%), quality of life (70\%), and maximum urinary flow rate (25\%). At the 12-month mark, improvements were sustained with regard to IPSS (41\%), quality of life $(60 \%)$, and maximum urinary flow rate $(32 \%)(\mathrm{p}<0.001)$. Patients did not report instances of erectile dysfunction or retrograde ejaculation. Thus, the authors concluded that not only is PUL effective in the treatment of BPH but also unique in that it can be performed under local anesthesia, an important notion in an aging population that may have several coexisting diseases supplementing prostatic enlargement.

With the advent and implementation of other minimally invasive technologies for the treatment of BPH (ex: Rezum), there is a strong need for comparison between these various surgical interventions. ${ }^{26}$ To this means, Tutrone et al compared outcomes between patients with non-retention BPH treated with PUL or Rezum (Tutrone). Fifty-three patients underwent Rezum $(n=23)$ or PUL $(n=30)$. There was no set exclusion for baseline symptoms, prostate size, or BPH medical therapy. Within 2 months postoperatively, patients completed questionnaires that captured patient experiences in several domains including urinary symptoms, recovery and interference with daily activities, treatment satisfaction and sexual function. Thereafter, outcomes were compared between the Rezum and PUL arms. ${ }^{26}$

Overall, despite the lack of exclusion criteria, patients included in each arm had similar baseline characteristics with respect to average age (PUL: 68 years old, SD 9.4 versus Rezum: 69 years old, SD 7.8), prostate size (PUL: 49 g, SD 28.4 versus Rezum: $63 \mathrm{~g}$, SD 30.9), and preoperative IPSS scores. In comparing outcomes following treatment with Rezum or PUL, patients who underwent PUL reported significantly improved IPSS scores and quality of life compared to those undergoing Rezum. Additionally, with regard to recovery and living a normal life post-procedure, PUL patients reported significantly less interference in daily activities, whether it be sports $(p=0.007)$, entertainment $(p=0.01)$, or in the community $(p=0.04)$, and although not statistically significant, PUL patients achieved a higher level of satisfaction with their treatment $(83 \%$ versus $65 \%$ for Rezum, $p=0.2)$. An important distinction to note is the duration of catheterization patients endure postoperatively; $7 \%$ of patients who underwent PUL were catheterized by postoperative day 3 compared to $55 \%$ of Rezum patients $(\mathrm{p}=0.0003)$. Finally, with regard to sexual function, SHIM scores were significantly higher in men who underwent PUL compared to those undergoing Rezum. Therefore, the findings of this study suggest that PUL provides a superior experience in multiple domains including improved sexual function, lower catheterization rates, higher patient satisfaction and less interference in daily life. ${ }^{26}$

On the other hand, a meta-analysis aiming to compare the newer minimally invasive treatments for BPH, evaluated four randomized-controlled trials reporting the outcomes after treatment. ${ }^{27}$ The authors found that with respect to urinary domain outcomes such as IPSS, peak flow rate, and PVR, ablative measures such as TURP and Aquablation showed greater improvement when compared to non-resective procedures such as Rezum and PUL. ${ }^{27}$ However, consistent with previous studies, patients who underwent PUL maintained a higher sexual function when compared to those undergoing TURP. Conversely to the work of Tutrone et al, the authors of this study did not experience a significant difference in urinary and sexual outcomes in patients undergoing PUL versus Rezum at two years of follow-up.

Finally, another meta-analysis and systematic review aimed to examine the sustainability of results (at least 24 
months of follow-up) in patients undergoing PUL. ${ }^{28}$ Five studies met the inclusion criteria, comprising a total of 386 patients, of which 322 patients $(83.4 \%)$ had at least 2-years follow-up. The authors categorized the studies as Group A and B according to whether the study was randomized or nonrandomized, respectively. Ultimately, at 24-months post-treatment, mean reduction in IPSS from baseline was 9.1 in Group $\mathrm{A}$ and 10.4 in Group B. Furthermore, patients in both groups experienced a similar improvement in peak flow rate and quality of life. With these findings, the authors further demonstrated the durability of PUL in terms of functional and sexual outcomes.

\section{Conclusion}

$\mathrm{BPH}$ is an exceedingly common condition affecting men. Lifestyle changes, medical management, and surgical interventions have been shown to improve outcomes for patients. Minimally invasive surgical methods such as PUL serve as a new and innovative means of treatment of LUTS. ${ }^{29}$ PUL has been shown to confer efficacious outcomes for patients experiencing LUTS, with results relatively comparable to those seen in TURP.

Patient selection is a critical component of electing PUL therapy, but recent studies have suggested expanding the range of patients who may be eligible for the procedure. Figure 1 shows a suggested pathway for determining whether patients

Patient with LUTS

attributed to BPH

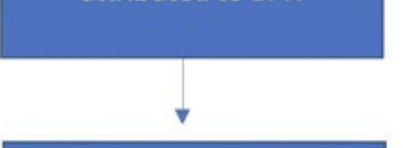

Symptoms refractory to

lifestyle changes and

pharmacological

management

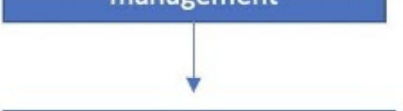

Consider surgical

intervention

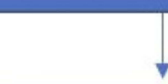

Prostate $<80 \mathrm{~g}$ with no

middle lobe obstruction or

patient with concern for

erectile and ejaculatory

function

Consider alternative

intervention such as

prostatectomy or laser

ablation

Figure I Suggested pathway to PUL for patients with LUTS secondary to BPH. 
may be suitable candidates for PUL (Figure 1). Some studies have shown that less symptomatic and older patients than those included in preliminary randomized trials may also benefit from PUL. In addition, preservation of sexual function continues to be an important benefit to PUL, and patients with concern for preserved ejaculatory and erectile function should be considered for PUL treatment. Further studies and particularly, long-term follow-up, and clinical trials are needed to optimize parameters for treatment of LUTS with PUL.

\section{Author Contributions}

All authors made substantial contributions to conception and design, acquisition of data, or analysis and interpretation of data; took part in drafting the article or revising it critically for important intellectual content; agreed to submit to the current journal; gave final approval of the version to be published; and agree to be accountable for all aspects of the work.

\section{Funding}

There is no funding to report.

\section{Disclosure}

The authors report no conflicts of interest in this work.

\section{References}

1. Lepor H. Pathophysiology, epidemiology, and natural history of benign prostatic hyperplasia. Rev Urol. 2004;6(Supp19):S3-s10.

2. Vuichoud C, Loughlin KR. Benign prostatic hyperplasia: epidemiology, economics and evaluation. Can J Urol. 2015;22(Suppl 1):1-6.

3. Robertson C, Link CL, Onel E, et al. The impact of lower urinary tract symptoms and comorbidities on quality of life: the $\mathrm{BACH}$ and UREPIK studies. BJU Int. 2007;99(2):347-354. doi:10.1111/j.1464410X.2007.06609.x

4. Novara G, Galfano A, Gardi M, Ficarra V, Boccon-Gibod L, Artibani W. Critical review of guidelines for BPH diagnosis and treatment strategy. Eur Urol Supplements. 2006;5(4):418-429. doi:10.1016/j.eursup.2006.02.005

5. Reich O, Gratzke C, Stief CG. Techniques and long-term results of surgical procedures for BPH. Eur Urol. 2006;49(6):970-978. doi:10.1016/j.eururo.2005.12.072

6. Emberton M, Fitzpatrick JM, Rees J. Risk stratification for benign prostatic hyperplasia (BPH) treatment. BJU Int. 2011;107(6):876-880. doi:10.1111/j.1464-410X.2010.10041.x

7. Geavlete P. Is classical transurethral resection of the prostate, the gold standard endoscopic treatment for benign prostate hyperplasia, in real danger of being replaced? Eur Urol. 2010;58(3):356. doi:10.1016/j. eururo.2010.06.001

8. Magistro G, Stief CG, Gratzke C. New intraprostatic injectables and prostatic urethral lift for male LUTS. Nat Rev Urol. 2015;12 (8):461-471. doi:10.1038/nrurol.2015.169

9. Pushkaran A, Stainer V, Muir G, Shergill IS. Urolift - minimally invasive surgical BPH management. Expert Rev Med Devices. 2017;14(3):223-228. doi:10.1080/17434440.2017.1296762
10. Jones P, Rai BP, Aboumarzouk O, Somani BK. UroLift: a new minimally-invasive treatment for benign prostatic hyperplasia. Ther Adv Urol. 2016;8(6):372-376. doi:10.1177/1756287216671497

11. Lebdai S, Chevrot A, Doizi S, et al. Do patients have to choose between ejaculation and miction? A systematic review about ejaculation preservation technics for benign prostatic obstruction surgical treatment. World J Urol. 2019;37(2):299-308. doi:10.1007/s00345018-2368-6

12. Bouhadana D, Nguyen DD, Zorn KC, Elterman DS, Bhojani N. Patient perspectives on benign prostatic hyperplasia surgery: a focus on sexual Health. J Sex Med. 2020;17(10):2108-2112.

13. Perera M, Roberts MJ, Doi SA, Bolton D. Prostatic urethral lift improves urinary symptoms and flow while preserving sexual function for men with benign prostatic hyperplasia: a systematic review and meta-analysis. Eur Urol. 2015;67(4):704-713. doi:10.1016/j.eururo.2014.10.031

14. Barkin J, Giddens J, Incze P, Casey R, Richardson S, Gange S. UroLift system for relief of prostate obstruction under local anesthesia. Can J Urol. 2012;19(2):6217-6222.

15. Haltbakk J, Hanestad BR, Hunskaar S. How important are men's lower urinary tract symptoms (LUTS) and their impact on the quality of life (QOL)? Qual Life Res. 2005;14(7):1733-1741. doi:10.1007/s11136-0053232-x

16. Roehrborn CG, Gange SN, Shore ND, et al. The prostatic urethral lift for the treatment of lower urinary tract symptoms associated with prostate enlargement due to benign prostatic hyperplasia: the. L I F T Study J Urol. 2013;190(6):2161-2167.

17. Roehrborn CG, Barkin J, Gange SN, et al. Five year results of the prospective randomized controlled prostatic urethral. L I F T Study Can J Urol. 2017;24(3):8802-8813.

18. Rukstalis D, Grier D, Stroup SP, et al. Prostatic Urethral Lift (PUL) for obstructive median lobes: 12 month results of the MedLift Study. Prostate Cancer Prostatic Dis. 2019;22(3):411-419. doi:10.1038/s41391-0180118-x

19. Shah BB, Tayon K, Madiraju S, Carrion RE, Perito P. Prostatic Urethral Lift: does size matter? J Endourol. 2018;32(7):635-638. doi:10.1089/end.2017.0855

20. Rukstalis D, Rashid P, Bogache WK, et al. 24-month durability after crossover to the prostatic urethral lift from randomised, blinded sham. BJU Int. 2016;118:14-22. doi:10.1111/bju.13666

21. Gratzke C, Barber N, Speakman MJ, et al. Prostatic urethral lift vs transurethral resection of the prostate: 2-year results of the BPH 6 prospective, multicentre, randomized study. BJU Int. 2017;119 (5):767-775. doi:10.1111/bju.13714

22. Al-Singary W, Patel R, Obi-Njoku O, Patel HRH. The UroLift System for lower urinary tract obstruction: patient selection for optimum clinical outcome. Minim Invasive Ther Allied Technol. 2020;1-6. doi:10.1080/13645706.2020.1816554

23. Sievert KD, Schonthaler M, Berges R, et al. Minimally invasive prostatic urethral lift (PUL) efficacious in TURP candidates: a multicenter German evaluation after 2 years. World $J$ Urol. 2019;37(7):1353-1360. doi:10.1007/s00345-018-2494-1

24. Eure G, Gange S, Walter P, et al. Real-World evidence of prostatic urethral lift confirms pivotal clinical study results: 2-year outcomes of a retrospective multicenter study. $J$ Endourol. 2019;33 (7):576-584. doi:10.1089/end.2019.0167

25. Kim JH, Lee KS, Kim TH. Evaluation of clinical outcomes of prostatic urethral lift for benign prostatic hyperplasia: an asian population study. World J Mens Health. 2020;38(3):338-344. doi:10.5534/wjmh.190015

26. Tutrone RF, Schiff W. Early patient experience following treatment with the UroLift prostatic urethral lift and Rezum steam injection. Can J Urol. 2020;27(3):10213-10219.

27. Tanneru K, Jazayeri SB, Alam MU, et al. An indirect comparison of newer minimally invasive treatments for benign prostatic hyperplasia: a network meta-analysis model. $J$ Endourol. 2021;35 (4):409-416. doi:10.1089/end.2020.0739 
28. Tanneru K, Gautam S, Norez D, et al. Meta-analysis and systematic review of intermediate-term follow-up of prostatic urethral lift for benign prostatic hyperplasia. Int Urol Nephrol. 2020;52(6):999-1008. doi:10.1007/s11255-020-02408-y
29. Magistro G, Stief CG, Woo HH. Mini-review: what is new in urolift? Eur Urol Focus. 2018;4(1):36-39. doi:10.1016/j.euf.2018.01.013

\section{Publish your work in this journal}

Research and Reports in Urology is an international, peer-reviewed, open access journal publishing original research, reports, editorials, reviews and commentaries on all aspects of adult and pediatric urology in the clinic and laboratory including the following topics: Pathology, pathophysiology of urological disease; Investigation and treatment of urological disease; Pharmacology of drugs used for the treatment of urological disease. The manuscript management system is completely online and includes a very quick and fair peer-review system, which is all easy to use. Visit http://www.dovepress.com/ testimonials.php to read real quotes from published authors. 